\title{
CARDIOVASCULAR SIGNAL RATE DETECTION USING FREQUENCY DOMAIN TRANSFORM
}

\author{
J. Hareen Breath Mary and C. Rekha \\ Department of Electronics and Communication Engineering, PET Engineering College, India
}

\begin{abstract}
Cardiovascular Rate (HR) is a crucial indicator of people's physiological state therefore it is important to monitor often. It is an essential parameter in most of the medical diagnosis for innumerable medical condition. This paper deals with the diagnosis of heart problems by computing the heart rate measurements from human facial videos recorded using a simple webcam. The main target is to extract cardiovascular rate from the skin color variation in the facial tissues caused due to oxygen supply all over the body. The face detection is carried out using Viola Jones algorithm which scans a subwindow capable of detecting faces across a given input image based on Haar-like rectangular features that are extracted from integral images. The forehead region is selected for Green channel estimation then Empirical mode decomposition is performed for reflectance decomposition of the forehead region. Finally, heart rate is estimated by detecting power spectral density of frequency domain images. Obtained PSD is compared with standard heart rate to obtain the subject's cardiovascular rate.
\end{abstract}

\section{Keywords:}

Viola Jones Algorithm, Haar-like Rectangular Feature, Reflectance Decomposition, Green Channel, PSD

\section{INTRODUCTION}

To diagnose the cardiovascular diseases by calculating the pulsation obtained from captured visage video using a simple camcorder. To attain a vigorous assessment, Intrinsic Mode functions is an excerpt to extract the cardiac rate to spot the cardiac diseases. Decomposition of reflectance of the face from a lone image to provide precise measurement. The problem definition is for detecting PQRST waves from ECG signal the present method uses traditional methods of ECG signal analysis using DSP. For different sets of data ICA and PCA must be conducted for extracting Heart Rate. Due to PCA's orthogonality there is no accurate information. Only by averaging these three methods (FFT, ICA, PCA) the better averaged results are obtained. The accuracy ranges with errors from $60 \%$ to $90 \%$. To avoid it empirical mode decomposition is performed.

Heart rate is achieved by absorbing skin color variations caused due to blood circulation throughout the body. As the heart beat varies, it changes the blood volume for each captured frame. Initially, the facial video is subjected to decomposition of face reflectance from a single image and from next consecutive frames. For examining the patient, the face is detected using Viola Jones algorithm and then an image covering the subject's brow area is selected for green channel estimation. Green channel is selected since only oxygenated blood absorbs green light, therefore green is selected among red and blue colors. Green channel involves reflectance decomposition. Ensemble empirical mode decomposition is used to extract IMF, which is further involved in estimation of heart disease.
Various electronic communication means are available, but only limited inconvenient options are available to the heart rate measurements to estimate heart disease. Therefore, to obtain heart rate, power spectral measurements are used using MATLAB software. Vital programming has written in MATLAB, appropriate software that estimates heart rate from the human facial video using Viola Jones face detection algorithm has been found to be a major learning resource.

\section{LITERATURE SURVEY}

Three different signal processing methods such as Fast Fourier Transform (FFT), Independent Component Analysis (ICA) and Principal Component Analysis (PCA) have been applied on the color channels in video recordings and the blood volume pulse (BVP) is extracted from the facial regions [2].

The Haar cascade classifier algorithm presented in Open CV is used to detect human face. By limiting a region of interest (ROI) in the face and extracting the raw RGB trace to a video frames in the ROI, independent component analysis (ICA) is applied to determine the strongest source signal used to estimate the pulse rate in beat per minute (PBM) [14].

The proposed strategy was thoroughly tried on the freely accessible by using PCA (Principle component analysis) algorithm with different techniques and the creators nearby dataset [1].

So a hefty individual visage video based cardiac rate supervising approach is exerted for the cardiac rate dissimilarity estimation, acquired without patient's physical contact.

Recognizing the visage from the input video mention to the identification of facial parts. Viola and Jones algorithm [16] has been implemented for this purpose, which uses Haar-like rectangular features that are extracted from integral images and display it as a rectangular box around the face detected which is directly used in our system. The most suitable parts for heart rate detection is the forehead region because all other regions except forehead region is subjected to no reflection due to the motion caused by heartbeat. Therefore, forehead region is used for processing.

Step 1: Capture the visage video using webcam.

Step 2: Visage Input video is displayed using MATLAB video player interface.

Step 3: RGB histogram is computed for every subsequent frames.

Step 4: Face is detected from visage video and its subsequent conversion into single frame and saving it as a separate image file.

Step 5: Forehead region is selected from visage image. 
Step 6: Extraction of green component is done for forehead region.

Step 7: Ensemble empirical mode decomposition is performed to extract IMF.

Step 8: Calculating heart rate based on PSD.

Step 9: The histogram displays for normal and abnormal PSD values.

Step 10: Heart disease identification based on comparison of normal with abnormal values.

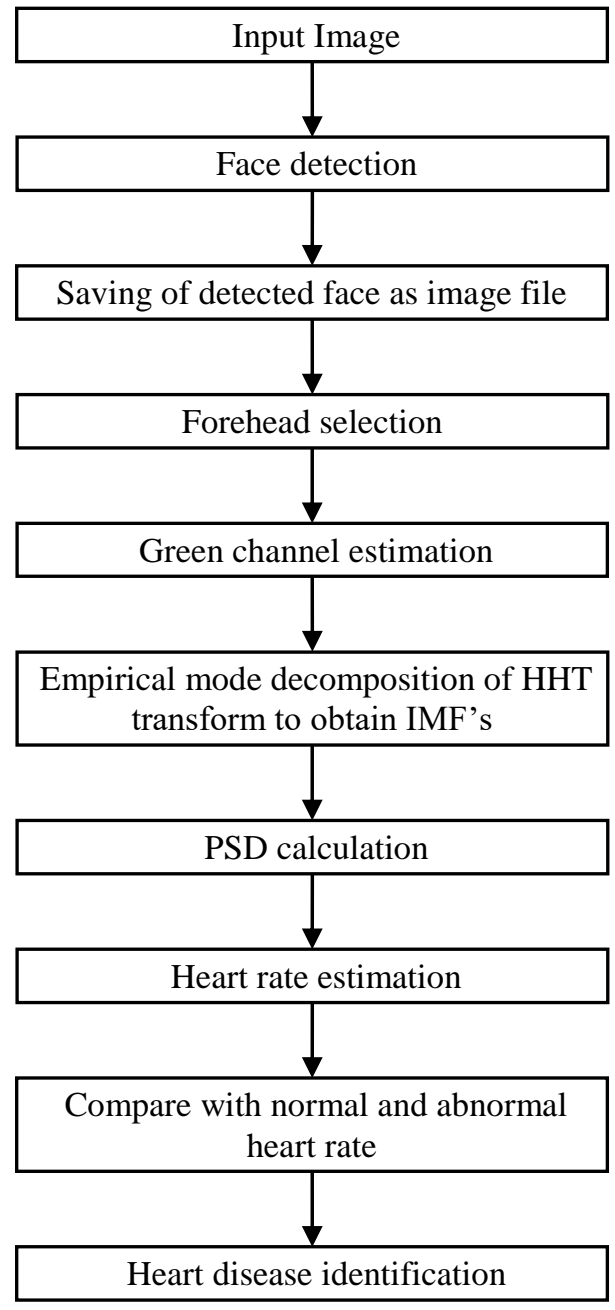

Fig.1. Overall block diagram

\subsection{VIOLA JONES ALGORITHM FOR VISAGE DETECTION}

The challenge to be solved is visage detection in an image. Humans can easily detect faces by looking into them, but a computer requires precise and concise instructions therefore Viola Jones is used. The target is to differentiate visage from nonvisage where identification is the primary step in the recognition of visage. The Viola jones algorithm is based on Haar Feature selection. For non-theoretical applications at least a pair of frames are processed for every second. The feature detection involves the grand total of picture element inside a rectangular area. All human visage has few similar properties of eyes, nose etc., these properties may be matched using Haar Features. Efficiency in visage detection is improved by generating an integral image first

$$
H(x, y)=\sum_{a=0}^{x} \sum_{b=0}^{y} X(a, b)
$$

Detection is performed within a rectangular box and each time a sliding step size is assumed. The window is slided over different scales and extract Haar features for visage detection.

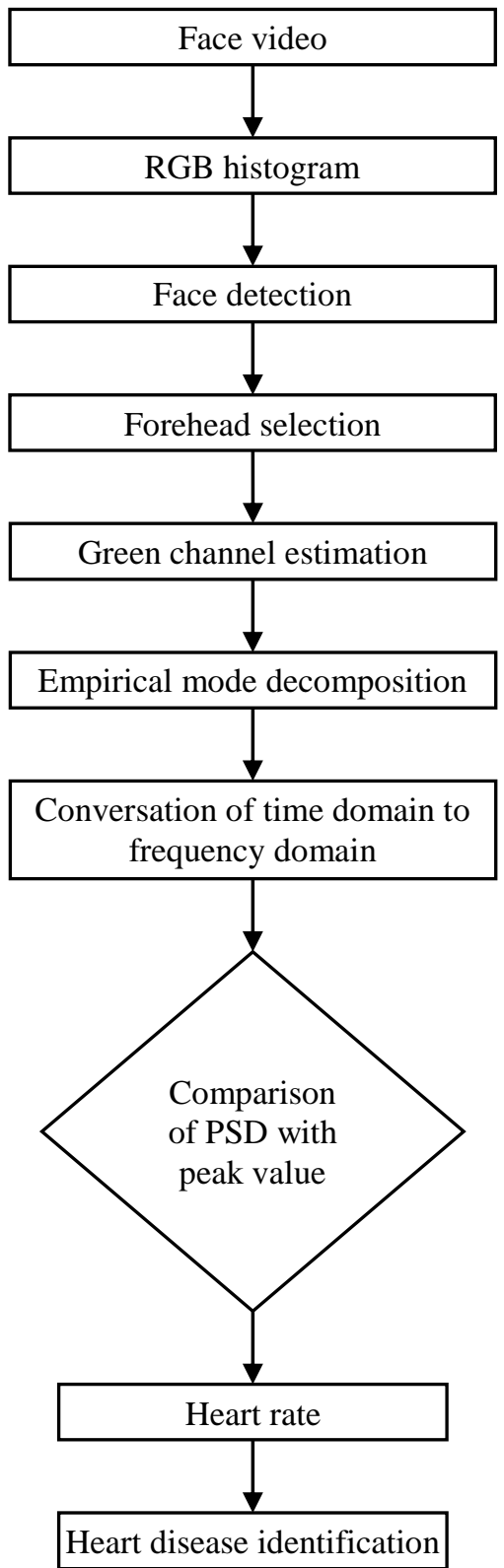

Fig.2. Flowchart of heart rate estimation

\subsection{HEART DISEASE IDENTIFICATION FROM HEART RATE DETECTION}

Major physiological signals measured in the human body is the Heart Rate which is carefully related to the overall health of a person. The heart rate is an indicator of the fitness levels of a person. Heart rate value differs for various physical activities such as exercising and even it changes while sleeping.

The absorption of oxygen by the body is directly proportional to the heart rate therefore it is checked constantly by medical 
professionals. Cardiac rate is described as heart contraction per minute i.e. throb of the heart

By analyzing the heart rate of a person using Intrinsic Mode Functions of Hilbert Huang's Transform the normal and abnormal heart rate values are compared. Based on the compared values of normal and abnormal heart rate, the presence of low blood pressure and depression state can be identified for the subjects under analysis

\section{RESULTS}

\subsection{INPUT VIDEO DISPLAYED USING MATLAB VIDEO PLAYER}

The Fig. 3 shows the video of pixel dimension $720 \times 1280$ which is given as input. The given input image is displayed using the video player interface. This video player initially performs building user interface for the video input and then initializing extensions operation is performed. Then rendering GUI is performed to load the input video then finally data source is loaded to display the video input.

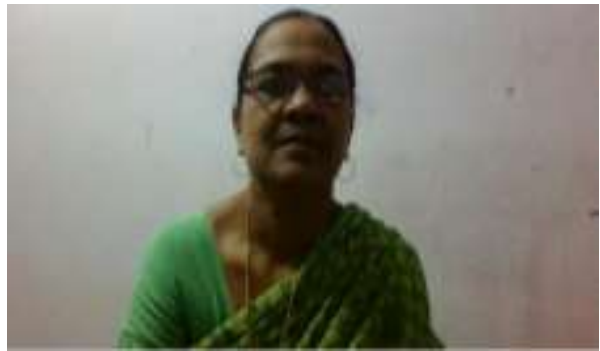

Fig.3. Input video

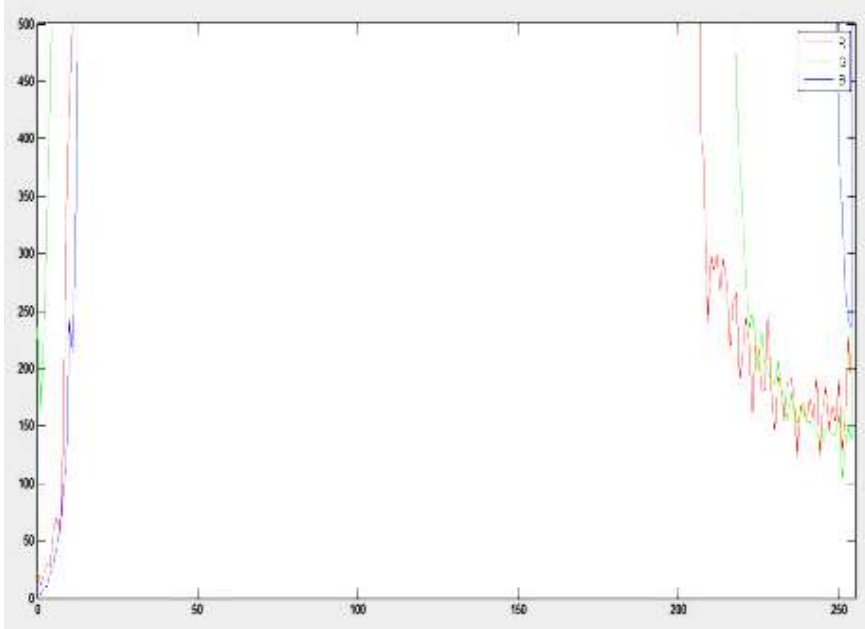

Fig.4. RGB Histogram for subsequent frames

The Fig. 4 shows the value of RGB varies and changes for each of the frames based on the reflectance. Since the reflectance value are represented as decomposition of face reflectance. Thus the face reflectance gets varied for each of the frames.

The $x$ axis ranges from 0 to 250 whereas $y$ axis varies from 0 to 500. The RGB value for each video frame is plotted in histograms for their variations. The loop through each color channel is estimated for frames $f_{1}, f_{2}, \ldots, f_{N}$. The RGB values for each frame is displayed in video histogram plots

\subsection{FACE DETECTION FROM VIDEO IMAGES AND CONVERSION OF VIDEO IMAGES INTO SINGLE FRAME}

The Fig.5 shows the input visage video of an individual. The video streams of the visage video are then converted into frames.

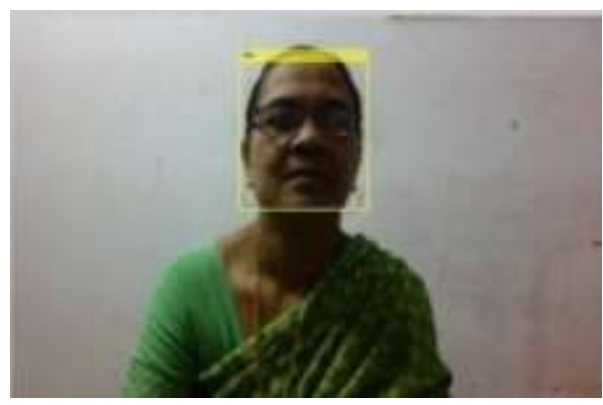

Fig.5. Face detected from input facial video

The Fig.6 shows the cropped face from single frame. The face detected using Viola Jones algorithm is stored at jpg format in the base directory by a counter function. The cropped image of the face are done to improve the accuracy in detecting the reflections due to blood flow throughout the body

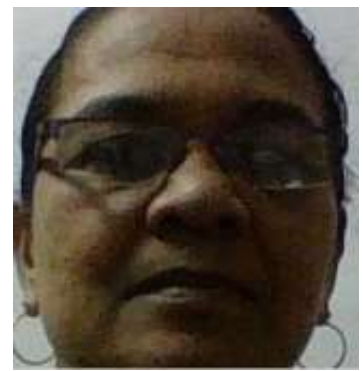

Fig.6. Cropped image of detected face

\subsection{FOREHEAD SELECTION}

The Fig.7 shows that the forehead region has low light absorption of the tissue and a good light reflection characteristic.

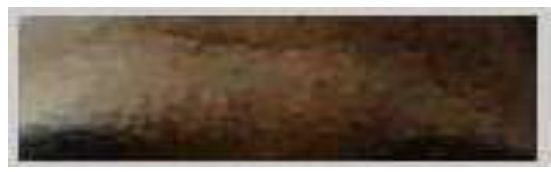

Fig.7. Selection of forehead area

Not all face regions are suitable for heart rate determination, because they do not provide necessary information due to strong movement artifacts, e. g. eyebrows, hair, eyes and mouth. Since forehead region disposes sufficient superficial vessels because of the thin skin, it was taken for analysis because region of interest (ROI) was selected to provide better quality and relatively low noise-affected heart rate.

\subsection{EXTRACTION OF GREEN COMPONENT}

Above the eyebrow region is chosen for green channel evaluation. 


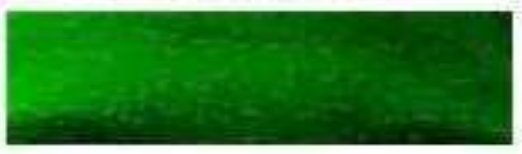

Fig.8. Green channel of eyebrow area

The Fig. 8 shows that the Green color is picked out among red and blue, because only oxygen carrying blood soak green. Green channel include reflectance decay.

\subsection{ENSEMBLE EMPIRICAL DECOMPOSITION}

MODE

Post processing as well as preprocessing are not demanded for Empirical mode decomposition (EMD). It is a data analysis technique to remove noise from non-linear and non-stationary processes.

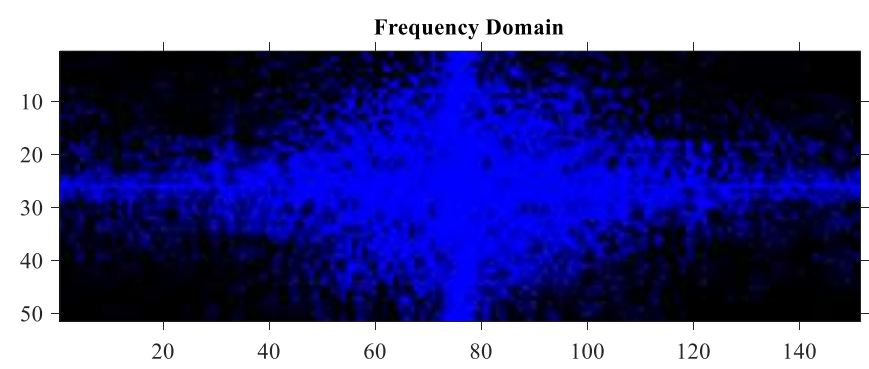

Fig.9. Frequency domain

The Fig.9 shows the conversion of time domain to frequency domain is performed using Fast Fourier Transform. Time domain is converted into frequency domain this is because the absorption level of light intensity is different for each frame. Therefore, there is a need for finding the Power Spectral Density (PSD) of each frame.

\subsection{CALCULATING HEART RATE}

The obtained heart rate using PSD calculations is compared with the normal heart rate $(1.2 \mathrm{~Hz})$ and approximated value is substituted in the standard equation. The real heart rate can be computed by the standard equation.

$$
H_{r}=\left(\phi^{*} F_{r} * 60\right) / O
$$

where, $\phi=$ maximum peak detected, $F_{r}=$ frame rate and $O$ is the total frames.

The Fig.10 shows the comparison of obtained heart rate of the subject with the abnormal heart rate to detect whether it is normal or abnormal, similarly it is also compared with normal heart rate to detect its normality of the subject's heart rate. The normal heart rate is the standard value for a person to be in normal state whereas the abnormal heart rate is the heart rate value lower or higher than the normal heart rate.
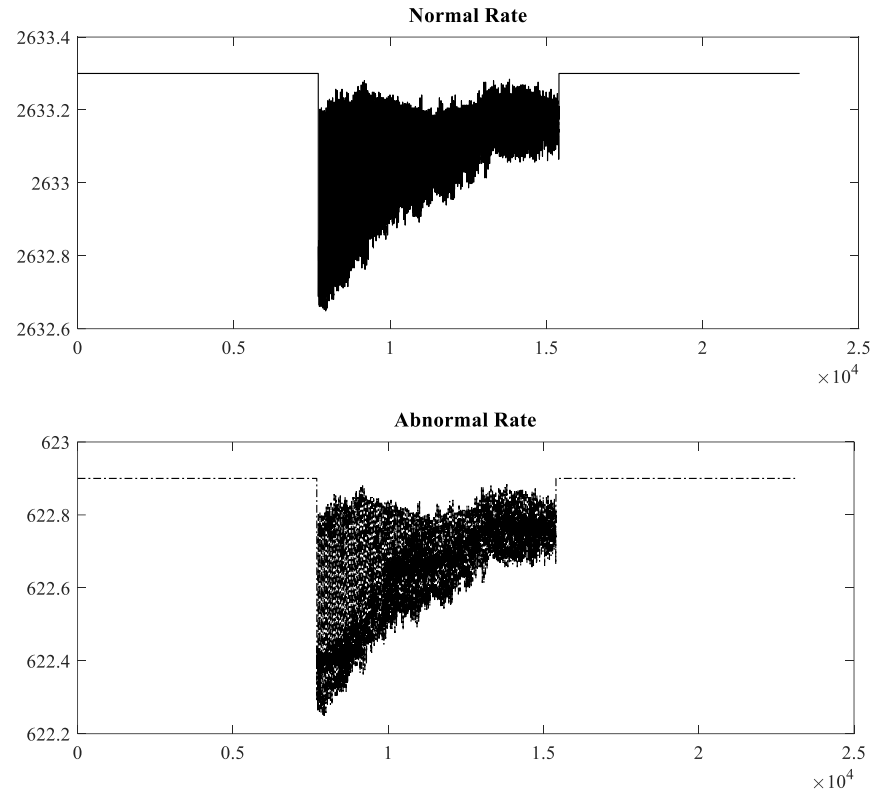

Fig.10. Individuals heart rate comparison from normal and abnormal heart rate

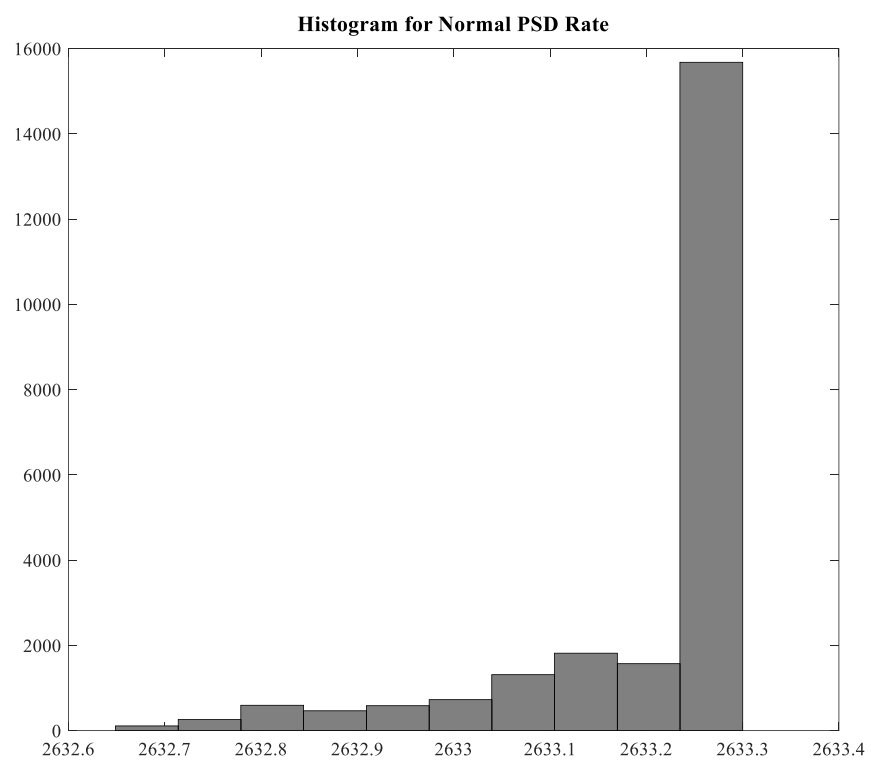

Fig.11. Histogram for normal PSD rate 


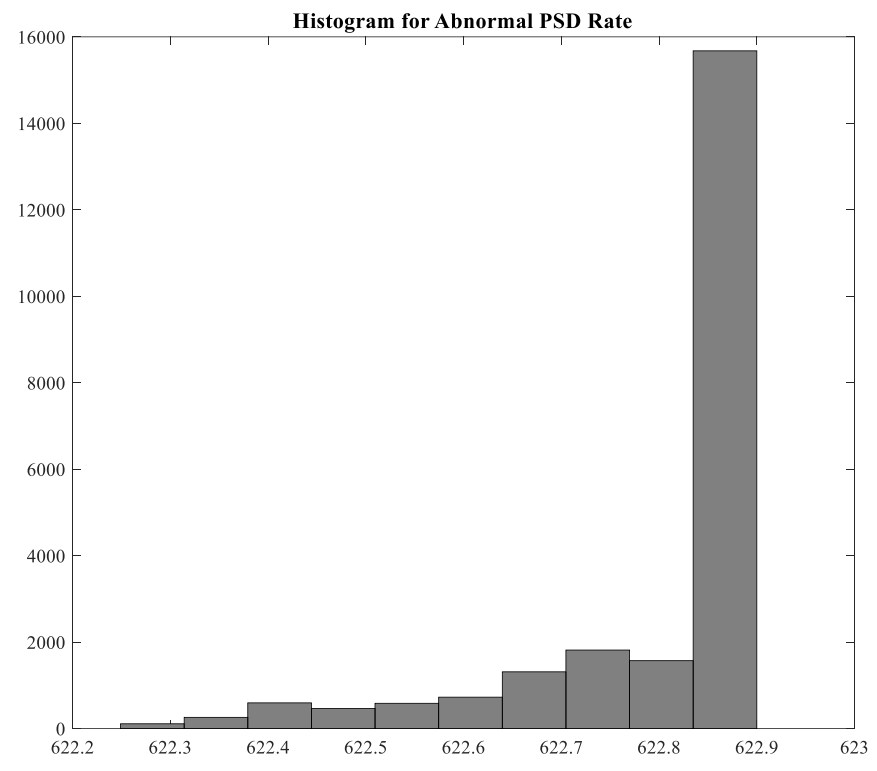

Fig.12. Histogram for abnormal PSD rate

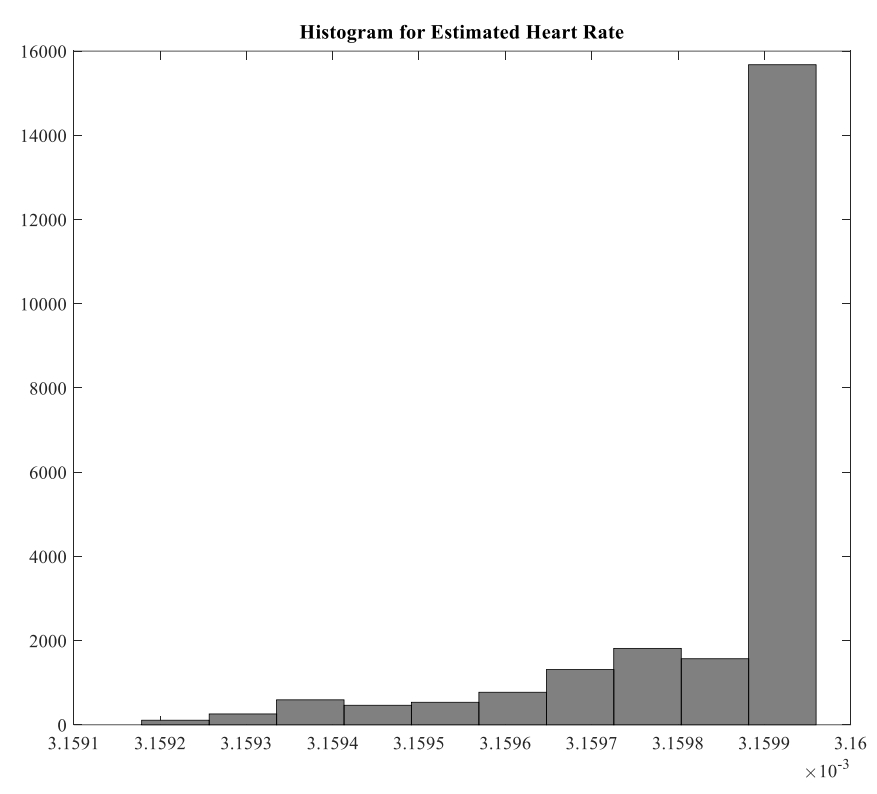

Fig.13. Histogram for estimated heart rate

The Fig.11 - Fig.13 shows the histogram display for normal and abnormal PSD values

\begin{tabular}{l|}
\hline INTRINSIC MODE FUNCTIONS \\
ans $=1.1486 \mathrm{e}+03$ \\
POWER SPECTRAL DENSITY \\
ans $=53577320$ \\
HEART RATE \\
ans $=64.2885$ \\
The person in the frame has no heart disease \\
\hline
\end{tabular}

Fig.14. Heart disease identification

The Fig.14 shows heart rate detection from PSD and disease identification. Depending on the correlation between normal and abnormal cardiac rate, the existence hypotension i.e., low blood pressure and melancholia can be distinguished for the visage video.

- Condition 1: if HR $\rightarrow 60<H_{r}<100 \rightarrow$ normal heart rate

- Condition 2: if HR $\rightarrow H_{r}<60 \rightarrow$ low blood pressure

- Condition 3: if HR $\rightarrow 100<H_{r} \rightarrow$ high blood pressure, the process is repeated for 3 to 4 times for every half an hour, if continues to be in high heart rate $\rightarrow$ presence of heart disease

Table.1. Comparison of heart rate of different age subjects

\begin{tabular}{|c|c|c|c|c|}
\hline Individuals & Age & IMF & PSD & Heart Rate \\
\hline Person 1 & 23 & $2.5220 \mathrm{e}+03$ & 60820604 & 72.9853 \\
\hline Person 2 & 22 & $2.2083 \mathrm{e}+03$ & 60819832 & 72.9850 \\
\hline Person 3 & 54 & $1.1486 \mathrm{e}+03$ & 53577320 & 64.2885 \\
\hline Person 4 & 70 & $1.8970 \mathrm{e}+03$ & 40826836 & 60.9880 \\
\hline
\end{tabular}

From Table.1, it is found that the heart rate varies depending upon the ages i.e., the heart rate for a person of age ranging from $22-23$ is $72 \mathrm{bpm}$ and for a person of higher age the heart rate is ranging from $60-64 \mathrm{bpm}$. The person with higher age also has low PSD.

\section{CONCLUSIONS}

Thus the heart rate analysis using Hilbert's Huang's transform for identifying heart disease was implemented and their performance were analyzed sufficiently and approximately. The proposed system would automatically estimate heart rate on the basis of power spectral density function and it would also perform efficient face detection using Viola Jones algorithm. This method provides highly accurate cardiovascular signal rate detection for disease identification. The experimental results were shown the guarantee and quiet simple process suitable for real time heart related applications and offline heart related applications.

In future work, the proposed framework will be developed with methodology to estimate heart related diseases based on the heart rate variations using multiple inputs of face video to provide vast improvements in real time applications. Intent to explore more observable features and several age classifications based on heart rate variations related to occurrence of heart diseases. Improvement of future work may also include improvement of intrinsic mode functions and thus increasing power spectral density.

\section{REFERENCES}

[1] Mohammad A. Haque, Ramin Irani, Kamal Nasrollahi and Thomas B. Moeslund, "Heart Beat Rate Measurement from Facial Video", IEEE Intelligent Systems, Vol. 31, No. 3, 2016.

[2] H. Rahman and M.U. Ahmed, S. Begum and P. Funk, "Real Time Heart Rate Monitoring from Facial RGB Color Video using Webcam", Proceedings of $29^{\text {th }}$ Annual Workshop of the Swedish Artificial Intelligence Society, pp. 1-8, 2016.

[3] Simmi Dutta, Hiteshwar, Abhimanyu Dev Jamwal and Azhar Ud Din Guroo, "Heart Rate Detection using Independent Component Analysis and Multivariate 
Adaptive Regression Splines", Imperial Journal of Interdisciplinary Research, Vol. 2, No. 10, 2016.

[4] M. Kumar, A. Veeraraghavan and A. Sabharwal, "Distance PPG: Robust Non-Contact Vital Signs Monitoring using A Camera", Biomedical Optics Express, Vol. 6, No. 5, pp. 1565-1588, 2015.

[5] Hussain A. Jaber, A.L. Ziarjawey and Ilyas Cankaya, "Heart Rate Monitoring and PQRST Detection Based on Graphical User Interface with Matlab", International Journal of Information and Electronics Engineering, Vol. 5, No. 4, pp. 311-317, 2015.

[6] J. Moreno, J. Ramos-Castro, J. Movellan, E. Parrado, G. Rodas and L. Capdevila, "Facial Video-based Photoplethysmography to Detect HRV at Rest", International Journal of Sports Medicine, Vol. 36, No. 6, pp. 474-480, 2015.

[7] Larissa Carvalho, H.G. Virani and S. Kutty, "Analysis of Heart Rate Monitoring Using a Webcam”, International Journal of Advanced Research in Computer and Communication Engineering, Vol. 3, No. 5, pp. 1-7, 2014.

[8] X. Li, J. Chen, G. Zhao, and M. Pietikainen, "Remote Heart Rate Measurement from Face Videos under Realistic Situations", Proceedings of IEEE International Conference on Computer Vision and Pattern Recognition, pp. 42644271, 2014.

[9] R. Irani, K. Nasrollahi and T.B. Moeslund, "Improved Pulse Detection from Head Motions using DCT", Proceedings of $9^{\text {th }}$ International Conference on Computer Vision Theory and Applications, pp. 124-129, 2014.
[10] S. Thulasi Prasad and S. Varadarajan, "Heart Rate Detection using Hilbert Transform", International Journal of Research in Engineering and Technology, Vol. 2, No. 8, pp. 12-18, 2013.

[11] Gerard De Haan and Vincent Jeanne, "Robust Pulse Rate from Chrominance-Based rPPG”, IEEE Transactions on Biomedical Engineering, Vol. 60, No. 10, pp. 94-128, 2013.

[12] G. Balakrishnan, F. Durand and J. Guttag, "Detecting Pulse from Head Motions in Video", Proceedings of IEEE International Conference on Computer Vision and Pattern Recognition, pp. 3430-3437, 2013.

[13] X. Yu, J. Huang, S. Zhang, W. Yan and D. Metaxas, "Posefree Facial Landmark Fitting Via Optimized Part Mixtures and Cascaded Deformable Shape Model", Proceedings of IEEE International Conference on Computer Vision and Pattern Recognition, pp. 1944-1951, 2013.

[14] Isayiyas Nigatu Tiba and Li Li, "Image-Based Automatic Pulse Rate Monitoring System Using PC Webcam", International Journal of Engineering Research and Technology, Vol. 2, No. 12, pp. 841-847, 2013.

[15] M. Soleymani, J. Lichtenauer, T. Pun and M. Pantic, "A Multimodal Database for Affect Recognition and Implicit Tagging", IEEE Transactions on Affective Computing, Vol. 3, No. 1, pp. 42-55, 2012.

[16] P. Viola and M. Jones, "Robust Real-time Object Detection", International Journal of Computer Vision, Vol. 4, No. 4, pp. 34-47, 2001. 